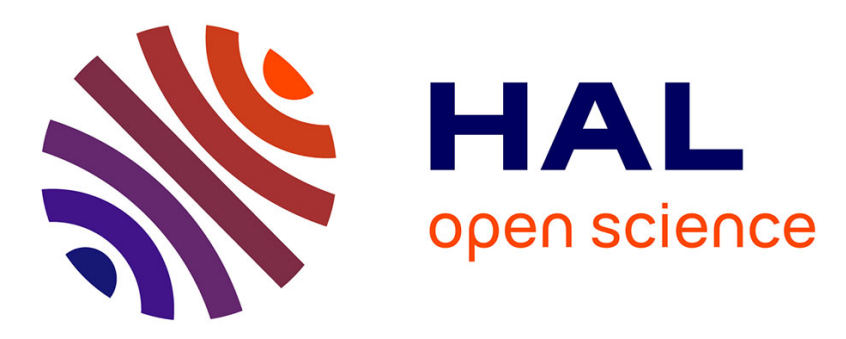

\title{
High-frequency leaky whispering-gallery modes in embedded elastic spheres
}

Fabien Treyssède, Matthieu Gallezot

\section{To cite this version:}

Fabien Treyssède, Matthieu Gallezot. High-frequency leaky whispering-gallery modes in embedded elastic spheres. Physical Review B, American Physical Society, 2021, 104 (21), 10.1103/PhysRevB.104.214101 . hal-03463333

\section{HAL Id: hal-03463333 \\ https://hal.archives-ouvertes.fr/hal-03463333}

Submitted on 2 Dec 2021

HAL is a multi-disciplinary open access archive for the deposit and dissemination of scientific research documents, whether they are published or not. The documents may come from teaching and research institutions in France or abroad, or from public or private research centers.
L'archive ouverte pluridisciplinaire HAL, est destinée au dépôt et à la diffusion de documents scientifiques de niveau recherche, publiés ou non, émanant des établissements d'enseignement et de recherche français ou étrangers, des laboratoires publics ou privés. 


\author{
High-frequency leaky whispering-gallery modes in embedded elastic spheres \\ Fabien Treyssède $\circledast^{1, *}$ and Matthieu Gallezot $\oplus^{1,2, \dagger}$ \\ ${ }^{1}$ GERS-GeoEND, Université Gustave Eiffel, IFSTTAR, F-44344 Bouguenais, France \\ ${ }^{2}$ Université de Nantes, Institut de Recherche en Génie Civil et Mécanique, BP 92208, Nantes, France
}

(Received 19 September 2021; accepted 22 November 2021; published xxxxxxxxxx)

\begin{abstract}
The goal of this paper is to investigate the characteristics of high-frequency whispering-gallery modes in embedded elastic spheres, that is, surrounded by an infinite elastic matrix. Due to several modeling difficulties, the high-frequency regime of embedded spheres has remained unexplored in elasticity. Our approach consists of formulating a specific finite-element method in spherical coordinates. The basic idea is to discretize only the radial coordinate while describing analytically the angular distribution of elastodynamic fields. Then, we also introduce a radial perfectly matched layer to cope with the unbounded nature of the external medium. Our approach yields a linear matrix eigensystem, simple and costless to solve. In order to identify general trends, both stiff and soft configurations are considered, corresponding to a sphere stiffer and softer than the external medium, respectively. Including material loss, our results highlight the behavior of leaky elastic whispering-gallery modes in the high-frequency regime. This work is motivated by the well-known behavior of whispering-gallery modes in optical resonators, reaching high $Q$ factors as the frequency increases. Identifying high- $Q$-factor whisperinggallery modes in elastic spheres could find promising applications for sensing the mechanical properties of external media.
\end{abstract}

DOI: 10.1103/PhysRevB.00.004100

\section{INTRODUCTION}

In this paper, we address the computation of leaky resonances of embedded elastic spheres at high acoustical frequencies. Our aim is to investigate the characteristics of whispering-gallery modes (WGMs) with particular attention to their attenuation ( $Q$ factor), which has not been considered yet. This work is motivated by the well-known behavior of WGMs in optical resonators [1], reaching high $Q$ factors as the frequency increases. High- $Q$-factor optical WGMs have sustained the development of numerous optical sensors [2]. Identifying such modes in elastic spheres could therefore find many interesting applications, e.g., for sensing the mechanical properties of a media external to the sphere.

Resonances of elastic spheres have been largely studied in vacuo, first by Lamb in 1881 as a classical problem in mechanics [3]. This topic has then drawn the attention of geophysicists, using the normal modes of the Earth to analyze the internal structure of our planet [4,5]. More recently, elastic resonances of nanoparticles have been studied because of their significant role in Raman scattering [6,7], stimulated Brillouin scattering [8], or surface plasmonic resonances [9].

However, the case of embedded elastic spheres, i.e., buried in an external solid matrix, is more intricate than in a vacuum. As a consequence, this case has been far less considered in the literature, and the analyses are most often limited to quite low acoustical frequencies [10-14]. Indeed, the modeling of

\footnotetext{
*fabien.treyssede@univ-eiffel.fr †pro@mgal.fr
}

an embedded elastic sphere can be described as an open resonator, which raises several difficulties.

First, the physics of open resonators strongly differ from closed systems: their spectrum involves a continuum of radiation modes, difficult to handle from a mathematical point of view. This continuum can be approximated by a discrete set, easier to manipulate, of so-called leaky modes (or quasinormal modes) [15]. Since energy leaks out of the sphere due to radiative loss, leaky resonances are damped in time: the eigenfrequencies are hence no longer real but necessarily complex. However, the behavior of leaky modes is somewhat unusual at infinity: while exponentially decreasing in time, these modes exponentially grow in the transverse direction $[15,16]$ (that is, in the radial direction for our spherical problem).

Second, radiative loss dramatically increases in a lowfrequency range, leading to a drastic drop of $Q$ factors. This motivates the consideration of high-frequency regimes, i.e., normalized frequency greater than $10^{2}$ in practice. However, high-frequency modes are difficult to solve by means of analytical methods because of the instabilities of secular equations $[14,17,18]$.

To circumvent this problem, our approach consists of formulating a specific finite-element (FE) method in spherical coordinates. Full three-dimensional or two-dimensional FE models are prohibited due to their computational cost in the high-frequency regime [19]. Therefore, the basic idea is to consider a semianalytical approach, consisting of separating the angular and the radial variables. The solution along the angular variables is analytical, decomposed on the basis of vector spherical harmonics. The solution along the radial variable is discretized, approximated with one-dimensional FE (see Fig. 1). This semianalytical FE principle was applied 


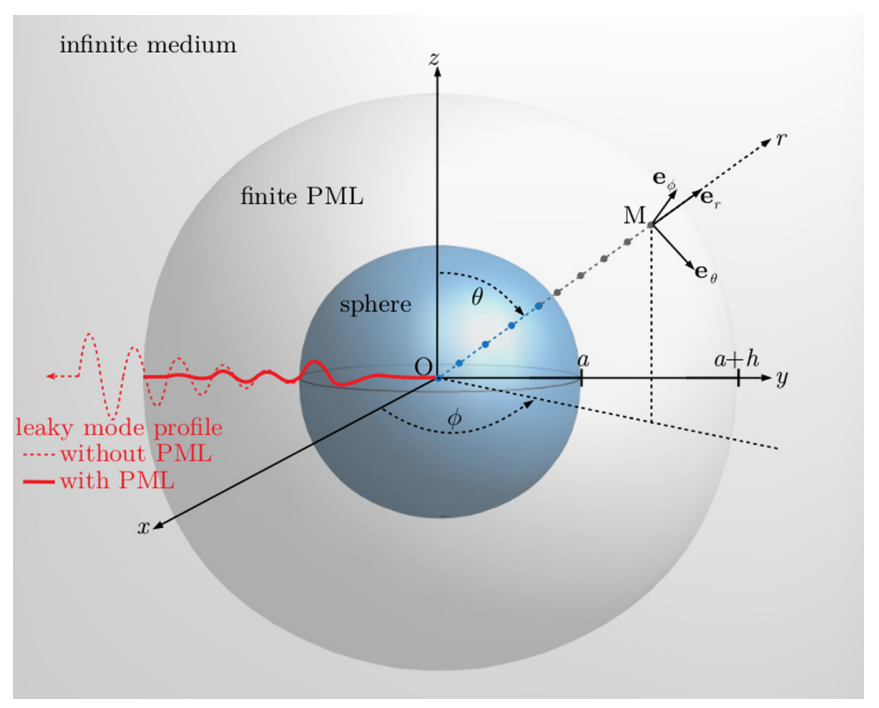

FIG. 1. Sphere of radius $a$ embedded into an infinite medium truncated with a PML of thickness $h$. The PML cancels the natural growth of leaky modes in the radial direction $r$, discretized by onedimensional FE (indicated by nodes). The spherical coordinates are the radius $r$, the polar angle $\theta(0 \leqslant \theta<\pi)$, and the azimuthal angle $\phi(0 \leqslant \phi<2 \pi)$. to compute the resonances of in vacuo spheres in [20,21], but without taking full advantage of the analytical description leading to tedious integral calculus. Recently, a compact formulation has been proposed in [22]. The main difficulty to achieve such a compact form in elasticity is to uncouple the spherical harmonics, which requires orthogonality relations of tensor type (the wave equation is vectorial) [23].

The goal of this paper is twofold: extend the formulation of Ref. [22] to the embedded case and highlight the behavior of leaky WGMs in the high-frequency regime. Nevertheless, a major difficulty for numerical methods is raised with the unbounded nature of the problem. This difficulty is particularly severe due to the exponential growth of leaky modes. A powerful technique to bound the problem consists in using a perfectly matched layer (PML). A PML is based on the complex scaling of the unbounded coordinate [24], which cancels the growth of leaky modes and allows truncating the unbounded embedding medium to a user-defined finite thickness (see Fig. 1). Regardless of spherical problems, this technique has been used to compute resonances in fluid and optical open cavities (see Refs. [25-28], for instance).

In this paper, we propose to combine a semianalytical FE formulation in spherical coordinates with a radial PML in order to compute leaky modes in embedded elastic spheres (Sec. II). Our approach yields a linear eigenproblem, which is very simple and costless to solve, even in the high-frequency regime (the FE discretization is one-dimensional). Compared to analytical approaches, our formulation does not suffer from instabilities and is quite versatile (complex inhomogeneous spheres, e.g., made of several layers, can be readily considered).

Last but not least, the formulation remains applicable for viscoelastic materials (complex elastic constants are allowed). The intrinsic loss of materials is usually far greater in elas- ticity than in optics. It is noteworthy that the effects of 114 viscoelastic loss tend to increase with frequency. Therefore, in the high-frequency regime, including viscoelastic loss in addition to leakage loss appears to be essential for a proper understanding of the physics of leaky WGMs in elasticity. Numerical results will be presented in Sec. III.

\section{SEMIANALYTICAL FE METHOD IN COMPLEX SPHERICAL COORDINATES}

\section{A. Elastodynamic weak form with radial PML}

Let us consider an elastic sphere of radius $a$ embedded into an infinite elastic matrix. With time-harmonic dependence $e^{-i \omega t}$, the displacement field $\tilde{\mathbf{u}}(r, \theta, \phi)=$ $\left[\tilde{u}_{r}(r, \theta, \phi), \tilde{u}_{\theta}(r, \theta, \phi), \tilde{u}_{\phi}(r, \theta, \phi)\right]^{\mathrm{T}} \quad(\mathrm{T}$ denotes matrix transpose) is the solution of the weak form of elastodynamics [29]:

$$
\int_{\tilde{V}} \delta \tilde{\boldsymbol{\epsilon}}^{\mathrm{T}} \tilde{\boldsymbol{\sigma}} d \tilde{V}-\omega^{2} \int_{\tilde{V}} \tilde{\rho} \delta \tilde{\mathbf{u}}^{\mathrm{T}} \tilde{\mathbf{u}} d \tilde{V}=0,
$$

with $d \tilde{V}=\tilde{r}^{2} d \tilde{r} \sin \theta d \theta d \phi$ in the spherical frame depicted in Fig. 1. Using Voigt notation, the stress and strain vectors are $\tilde{\boldsymbol{\sigma}}=\left[\tilde{\sigma}_{r r}, \tilde{\sigma}_{\theta \theta}, \tilde{\sigma}_{\phi \phi}, \tilde{\sigma}_{\theta \phi}, \tilde{\sigma}_{r \phi}, \tilde{\sigma}_{r \theta}\right]^{\mathrm{T}}$ and $\tilde{\boldsymbol{\epsilon}}=$ $\left[\tilde{\epsilon}_{r r}, \tilde{\epsilon}_{\theta \theta}, \tilde{\epsilon}_{\phi \phi}, 2 \tilde{\epsilon}_{\theta \phi}, 2 \tilde{\epsilon}_{r \phi}, 2 \tilde{\epsilon}_{r \theta}\right]^{\mathrm{T}}$. The stress-strain relationship is $\tilde{\boldsymbol{\sigma}}=\tilde{\mathbf{C}} \tilde{\boldsymbol{\epsilon}}$, where $\tilde{\mathbf{C}}$ is the matrix of material properties. The external medium is assumed as homogeneous and isotropic. The sphere can be transversely isotropic and inhomogeneous (e.g., constituted by several layers). For transversely isotropic materials, the matrix depends on five independent coefficients and can be written as

$\tilde{\mathbf{C}}=\left[\begin{array}{cccccc}C_{11} & C_{12} & C_{12} & 0 & 0 & 0 \\ C_{12} & C_{23}+2 C_{44} & C_{23} & 0 & 0 & 0 \\ C_{12} & C_{23} & C_{23}+2 C_{44} & 0 & 0 & 0 \\ 0 & 0 & 0 & C_{44} & 0 & 0 \\ 0 & 0 & 0 & 0 & C_{55} & 0 \\ 0 & 0 & 0 & 0 & 0 & C_{55}\end{array}\right]$.

The strain-displacement relationship is $\tilde{\boldsymbol{\epsilon}}=\tilde{\mathbf{L}} \tilde{\mathbf{u}}$ with

$$
\tilde{\mathbf{L}}=\mathbf{L}_{r} \frac{\partial}{\partial \tilde{r}}+\mathbf{L}_{\theta} \frac{\partial}{\tilde{r} \partial \theta}+\mathbf{L}_{\phi} \frac{\partial}{\tilde{r} \sin \theta \partial \phi}+\frac{1}{\tilde{r}} \mathbf{L}_{1}+\frac{\cot \theta}{\tilde{r}} \mathbf{L}_{2},
$$

where

$$
\begin{aligned}
\mathbf{L}_{r} & =\left[\begin{array}{lll}
1 & 0 & 0 \\
0 & 0 & 0 \\
0 & 0 & 0 \\
0 & 0 & 0 \\
0 & 0 & 1 \\
0 & 1 & 0
\end{array}\right], \mathbf{L}_{\theta}=\left[\begin{array}{lll}
0 & 0 & 0 \\
0 & 1 & 0 \\
0 & 0 & 0 \\
0 & 0 & 1 \\
0 & 0 & 0 \\
1 & 0 & 0
\end{array}\right], \mathbf{L}_{\phi}=\left[\begin{array}{lll}
0 & 0 & 0 \\
0 & 0 & 0 \\
0 & 0 & 1 \\
0 & 1 & 0 \\
1 & 0 & 0 \\
0 & 0 & 0
\end{array}\right], \\
\mathbf{L}_{1} & =\left[\begin{array}{ccc}
0 & 0 & 0 \\
1 & 0 & 0 \\
1 & 0 & 0 \\
0 & 0 & 0 \\
0 & 0 & -1 \\
0 & -1 & 0
\end{array}\right], \mathbf{L}_{2}=\left[\begin{array}{ccc}
0 & 0 & 0 \\
0 & 0 & 0 \\
0 & 1 & 0 \\
0 & 0 & -1 \\
0 & 0 & 0 \\
0 & 0 & 0
\end{array}\right] .
\end{aligned}
$$

Along the radius, we introduce a radial PML thanks to ${ }_{141}$ an analytic continuation [24] of the weak form (1) into the 142 
complex transverse coordinate $\tilde{r}$ :

$$
\tilde{r}(r)=\int_{0}^{r} \gamma(\xi) d \xi
$$

The function $\gamma(r)$ is a user-defined function, with $\operatorname{Im} \gamma(r)>$ 0 in the PML region, which enables the absorption of outgoing waves in the embedding medium. The PML is truncated to a thickness $h$ to obtain a reflectionless bounded problem. In this paper, we choose a parabolic attenuation profile:

$$
\gamma(r)=\left\{\begin{array}{cl}
1, & \text { if } r \leqslant a, \\
1+3(\hat{\gamma}-1)\left(\frac{r-d}{h}\right)^{2}, & \text { if } \quad r>a .
\end{array}\right.
$$

The PML interface is set at $r=a$; that is, stick to the physical interface between the sphere and the embedding medium, to avoid the computation of spurious eigenvalues [26]. For convenience, we define the parameter $\hat{\gamma}=\frac{1}{h} \int_{d}^{d+h} \gamma(\xi) d \xi$, the user-defined averaged value of $\gamma(r)$ inside the PML. At the end of the PML $(r=a+h)$, a Dirichlet boundary condition is applied.

Finally, the weak form (1) can be transformed to go back to the real radial direction $r$ thanks to the following change of variable $\tilde{r} \mapsto r$, for any function $\tilde{g}(r)$ :

$$
\begin{aligned}
\tilde{g}(\tilde{r}) & =g(r), d \tilde{r}=\gamma(r) d r, \\
\frac{\partial \tilde{g}}{\partial \tilde{r}} & =\frac{1}{\gamma(r)} \frac{\partial g}{\partial r} .
\end{aligned}
$$

\section{B. Semianalytical FE formulation}

Since the problem has been bounded by a finite PML, we can now apply the same procedure as in Ref. [22] to obtain a semianalytical FE formulation. For brevity, we recall the main steps of the procedure in the following (readers are invited to see Ref. [22] for further details).

The basic idea is to treat analytically the angular behavior of the solution and to use numerical discretization along the radial coordinate. Based on Refs. [30,31], the displacement field can be decomposed into vector spherical harmonics:

$$
\mathbf{u}(r, \theta, \phi)=\sum_{l \geqslant 0} \sum_{|m| \leqslant l} \mathbf{S}_{l}^{m}(\theta, \phi) \hat{\mathbf{u}}_{l}^{m}(r) .
$$

The matrix $\mathbf{S}_{l}^{m}(\theta, \phi)$ concatenates the vector spherical harmonics which describe the angular distribution of the three components of the displacement field. This matrix is explicitly given by

$$
\mathbf{S}_{l}^{m}(\theta, \phi)=\left[\begin{array}{ccc}
\mathrm{Y}_{l}^{m}(\theta, \phi) & 0 & 0 \\
0 & \frac{\partial Y_{l}^{m}(\theta, \phi)}{\partial \theta} & -\frac{\partial Y_{l}^{m}(\theta, \phi)}{\sin \theta \partial \phi} \\
0 & \frac{\partial Y_{l}^{m}(\theta, \phi)}{\sin \theta \partial \phi} & \frac{\partial Y_{l}^{m}(\theta, \phi)}{\partial \theta}
\end{array}\right],
$$

where $Y_{l}^{m}(\theta, \phi)$ corresponds to normalized spherical harmonics of integer degree $l$ and order $m(|m| \leqslant l)$ [32]. $l$ and $m$ are also called polar and azimuthal wave numbers, respectively. The vector $\hat{\mathbf{u}}_{l}^{m}(r)=\left[\hat{u}_{l}^{m}(r), \hat{v}_{l}^{m}(r), \hat{w}_{l}^{m}(r)\right]^{\mathrm{T}}$ is the $(l, m)$ coefficient of the vector spherical harmonic transform of the physical field $\mathbf{u}(r, \theta, \phi)$. With a full analytical method, these coefficients could be expressed as spherical Bessel functions (for the interior problem with $r \leqslant a$ ) and spherical Hankel functions (for the exterior problem with $r>a$ ) [17,31]. Instead, a one-dimensional FE approximation is used in this

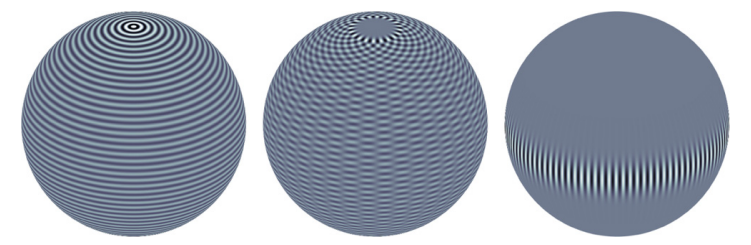

FIG. 2. Normal displacement distribution for the following spherical harmonics (from left to right): $(l, m)=(100,0),(l, m)=$ $(100,20),(l, m)=(100,100)$, corresponding to zonal, tesseral, and sectoral patterns, respectively (WGMs are of sectoral type).

paper, so that

$$
\hat{\mathbf{u}}_{l}^{m, e}(r)=\mathbf{N}^{e}(r) \hat{\mathbf{U}}_{l}^{m, e},
$$

where $\mathbf{N}^{e}(r)$ is the matrix of interpolating functions and $\hat{\mathbf{U}}_{l}^{m, e}$ is the vector of degrees of freedom (dofs) at the element level (e).

To achieve the separation of angular and radial variables, the key point is to properly choose the arbitrary test fields. We choose the virtual displacements as

$$
\delta \mathbf{U}^{e \mathrm{~T}}(r, \theta, \phi)=\delta \hat{\mathbf{U}}^{e \mathrm{~T}} \mathbf{N}^{e \mathrm{~T}}(r) \mathbf{S}_{k}^{p *}(\theta, \phi),
$$

where the operation * stands for transpose conjugate. Thanks to this choice, it can be shown that the orthogonality relations of both vector [31] and tensor [23] spherical harmonics appear in Eq. (1) when integrating over the angular coordinates (see Ref. [22] for details). This choice is therefore fundamental to get uncoupled equations for every spherical harmonics $(l, m)$ of the displacement field. After lengthy calculations, the following compact matrix system is obtained for a given pair $(l, m)$ :

$$
\left[\mathbf{K}(l)-\omega^{2} \mathbf{M}(l)\right] \hat{\mathbf{U}}_{l}^{m}=\mathbf{0} .
$$

The detailed expressions of the elementary stiffness and mass matrices are given in Appendix. Equation (12) is a linear eigenproblem with respect to $\omega^{2}$. For a given value of $l$, it yields $n=1, \ldots, N$ eigenfrequencies $\omega_{l}^{(n)}$ and eigenvectors $\hat{\mathbf{U}}_{l}^{(n)}$. The latter corresponds to radial mode shapes.

Regarding analytical approaches $[17,30]$, two main features are recovered by the matrix eigensystem (12). First, the eigensystem can be subdivided into two independent linear eigenproblems corresponding on the one hand to spheroidal modes (with nonzero displacements in each direction), and on the other hand to torsional modes (with zero displacement in the radial direction). This can be easily deduced from the structure of matrices in Eqs. (A1)-(A5).

Second, it can be noticed that the eigensystem is degenerate with respect to the azimuthal wave number $m$. This means that $2 l+1$ modes have the same eigenfrequency and the same radial mode shape [33]. These multiple modes only differ from their angular distribution.

Figure 2 shows the angular distribution of the normal displacement $\hat{u}_{l}^{m}$, given by $Y_{l}^{m}(\theta, \phi)$ [see Eq. (9)], for three spherical harmonics: $(l, m)=(100,0),(l, m)=(100,20)$, and $(l, m)=(100,100)$. The distributions corresponding to $m=0, m \neq l$, and $m=l$ are the zonal, tesseral, and sectoral patterns of spherical harmonics, respectively $[31,32]$. As observed in Fig. 2, sectoral modes are confined at the equator 
$(\theta=\pi / 2)$ and are the so-called WGMs, which have found many applications in optics $[1,2,34]$. Note that the distribution of WGMs gets narrower near the equator for increasing wave numbers $l=m$. This means that narrow WGMs can be achieved only at a high-frequency regime in practice.

\section{Improved conditioning for large wave number}

With Eq. (12), ill conditioning may result for large value of $l$. This can be easily deduced from the expression of the mass matrix, given by Eq. (A1), where terms of order 1 and $l^{2}$ occur together in the diagonal inner matrix. To circumvent this problem, we introduce the following transformation for $l \neq 0$ :

$$
\hat{\mathbf{u}}_{l}^{m}=\left[\begin{array}{ccc}
1 & 0 & 0 \\
0 & \frac{1}{\sqrt{\bar{l}}} & 0 \\
0 & 0 & \frac{1}{\sqrt{l}}
\end{array}\right] \hat{\boldsymbol{\phi}}_{l}^{m},
$$

which merely consists in dividing by $\sqrt{\bar{l}}$ the angular components of the transformed displacement field, denoted as $\hat{\boldsymbol{\phi}}_{l}^{m}$.

At the global FE level, we denote this transformation as follows:

$$
\hat{\mathbf{U}}_{l}^{m}=\mathbf{T}(l) \hat{\boldsymbol{\Phi}}_{l}^{m}
$$

where $\mathbf{T}(l)$ is the global transformation matrix. Then, the initial eigensystem (12) is transformed into

$$
\left(\mathbf{T}^{\mathrm{T}} \mathbf{K} \mathbf{T}-\omega^{2} \mathbf{T}^{\mathrm{T}} \mathbf{M} \mathbf{T}\right) \hat{\boldsymbol{\Phi}}_{l}^{m}=\mathbf{0},
$$

where the dependence on $l$ of matrices has been dropped for conciseness of notations.

With this transformation, it can be checked that the inner diagonal terms of the element mass matrix (A1) are now all transformed to unity. The eigensystem (15) hence remains well conditioned for large $l$. Note also that the symmetry of the eigensystem has been preserved.

\section{Spectrum characteristics}

As outlined in the introduction, the physics of open resonators is significantly different from in vacuo systems. Understanding their spectral behavior is essential to properly exploit the numerical results.

We define $k_{l, s_{\infty}}^{2}=\omega^{2} / c_{l, s_{\infty}}^{2}$, the shear and longitudinal wave numbers in the infinite medium. Let us first briefly recall some theoretical results by considering the unbounded problem without introducing any PML. Theoretically, the spectrum of an unbounded problem is constituted by a continuum of radiation modes and proper discrete poles $[15,35-$ 37].

Proper discrete poles are referred to as trapped modes, having a pure real resonance frequency if material loss is neglected (infinite $Q$ factor). They are located on the Riemann sheet $\operatorname{Im}\left(k_{l, s_{\infty}}\right) \geqslant 0$, where fields spatially decay at infinity. The occurrence of trapped modes depends on the configuration of the problem. Actually, such modes never exist in spherical problems. This can be shown using Rellich's lemma [38], stating that if the energy is zero far from the sphere then the displacement is zero everywhere (that is, only the zero eigenvalue satisfies the decaying wave condition at infinity).

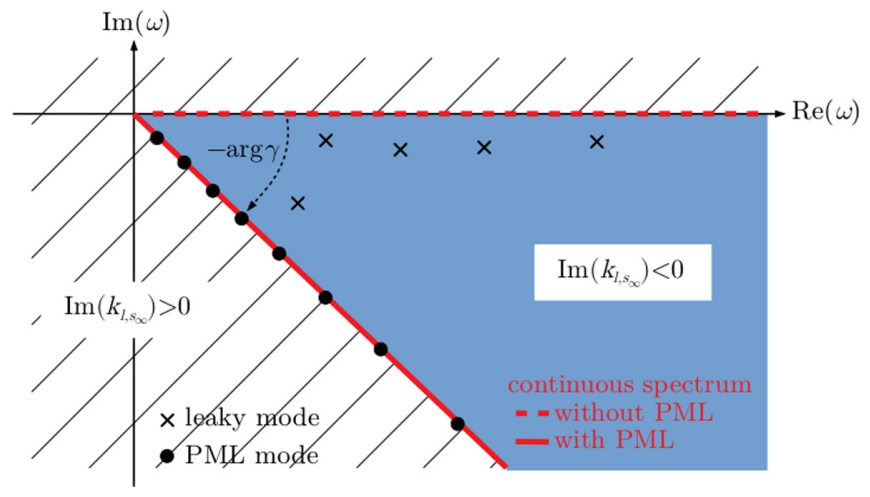

FIG. 3. Representation of the eigenspectrum of an open resonator in the complex frequency plane. Red dashed line: Continuum of radiation modes without PML [branch cuts $\left.\operatorname{Im}\left(k_{l, s_{\infty}}\right)=0\right]$. Red solid line: Continuum rotated by the infinite PML [branch cuts $\left.\operatorname{Im}\left(\gamma k_{l, s_{\infty}}\right)=0\right]$. Blue region: Portion of the initial improper Riemann sheets, $\operatorname{Im}\left(k_{l, s_{\infty}}\right)<0$, accessible thanks to the PML rotation and containing leaky modes (crosses). Black bullets indicate PML modes (discretization of the continuum caused by truncation of PML to a finite thickness).

This a major difference with flat problems, for which trapped waves can occur (such as Stoneley waves [39]).

As a consequence, only the continuum of radiation modes theoretically takes part in the solution of our problem. For elastic waves, this continuum corresponds to the contribution of two branch cuts given by $\operatorname{Im}\left(k_{l, s_{\infty}}\right)=0$. These two branch cuts define a fourfolded Riemann surface for the square roots of $k_{l, s_{\infty}}^{2}$ (multivalued functions), according to the sign of $\operatorname{Im}\left(k_{l_{\infty}}\right)$ and $\operatorname{Im}\left(k_{s_{\infty}}\right)$. Figure 3 depicts the branch cuts in the complex frequency plane. Both branch cuts coincide with each other for pure real bulk wave speeds $c_{l, s_{\infty}}$ (in the case of viscoelastic materials, the imaginary part of wave speeds is yet relatively small compared to the real part so that both branch cuts remain almost coincident in practice).

Actually, a second type of discrete mode occurs, corresponding to complex-valued poles located in the improper Riemann sheets [i.e., $\operatorname{Im}\left(k_{l_{\infty}}\right)<0$ and/or $\operatorname{Im}\left(k_{s_{\infty}}\right)<0$ ]. These improper eigenvalues are the so-called leaky modes (or quasinormal modes). They do not satisfy the spatially decaying wave condition at infinity, and hence, do not theoretically contribute to the exact solution. However, leaky modes can be conveniently used to approximate the continuum of radiation modes as a discrete sum. Besides, they reveal key information, hidden inside the continuum, about wave properties (group velocity, attenuation, etc.) [40]. Note that, because trapped modes do not exist in spherical problems, the WGMs of embedded spheres are indeed necessarily leaky modes.

Now, let us introduce an infinite PML. As demonstrated in Refs. [26,37], this further modifies the eigenspectrum. For simplicity, we assume a constant attenuation function $\gamma$ inside the PML. Introducing an infinite PML changes the branch cuts to $\operatorname{Im}\left(\gamma k_{l, s_{\infty}}\right)=0$. This corresponds to a branch-cut rotation in the complex-frequency plane by the angle of rotation $-\arg \gamma$ (see Fig. 3). With a PML, the proper Riemann surface is now given by $\operatorname{Im}\left(\gamma k_{l, s_{\infty}}\right) \geqslant 0$. This surface contains leaky modes, revealed by the branch-cut rotation (blue region in 


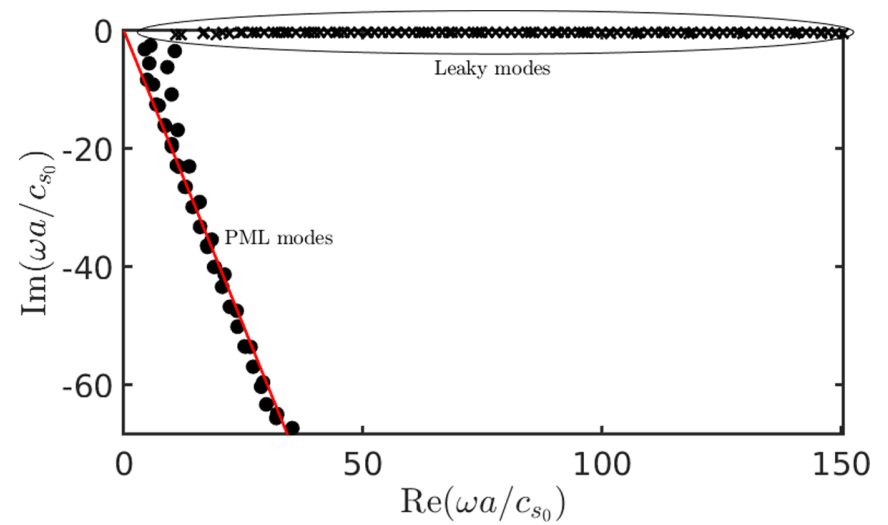

FIG. 4. Spectrum computed for a steel sphere into concrete $(l=10, \hat{\gamma}=1+2 i, h=0.25 a)$. Black bullets: PML modes; black crosses: leaky modes identified from the criterion given by Eq. (16). Red line: Theoretical branch cut (rotated by $-\arg \hat{\gamma}$ ).

Fig. 3). The number of revealed leaky resonances depends on the argument of $\gamma$.

Finally, let us truncate the infinite PML to a finite thickness $h$. Truncation induces discretization of branch cuts: the continuum of radiation modes is transformed into a discrete set of radiation modes [26,41], with finer discretization as the PML thickness increases $[15,27,40]$. These modes are often called PML modes (see Fig. 3). They resonate mainly inside the PML and are hence not intrinsic to the physics (they strongly depend on the user-defined PML parametrization). Although a solution of the eigenvalue problem, PML modes are therefore of little interest for the analysis of this paper.

\section{E. Mode filtering}

As a postprocessing step, it is necessary to filter out PML modes from the solution in order to properly visualize only the leaky WGMs. Following Refs. [42,43], leaky modes can be identified efficiently from the following energy-based criterion:

$$
1-\frac{\operatorname{Im}\left(E_{l}^{m}\right)}{\left|E_{l}^{m}\right|}>\eta_{\min },
$$

where $\eta_{\min }$ is a user-defined threshold and $E_{l}^{m}=$ $\frac{1}{2}\left|\omega_{l}^{2}\right| \hat{\mathbf{U}}_{l}^{m *} \mathbf{M} \hat{\mathbf{U}}_{l}^{m} . E_{l}^{m}$ can be interpreted as the kinetic energy integrated over the radius, including the PML. The imaginary part of this energy is expected to be much greater inside the PML for PML modes than for leaky resonances (which are expected to resonate mostly inside the sphere).

As an example, Fig. 4 shows a typical spectrum computed with the semianalytical FE formulation. The frequency is normalized as $\omega a / c_{s_{0}}$, where $c_{s_{0}}$ denote the shear bulk wave velocity of the sphere material. The test case, a steel sphere buried into concrete, is described later in the next section. As explained earlier at the end of Sec. II B, the whole spectrum consists of two separate sets of modes, spheroidal and torsional modes (both types of modes have been computed together and are not distinguished in the figure). The latter are related to shear waves only [44] and give only one continuous set. The former are a mixture of longitudinal and shear waves [44] and give two continuous sets. As observed in Fig. 4, these continuous sets are discretized by the finite PML, yielding PML modes which are close to the theoretical branch cuts (rotated by $-\arg \hat{\gamma}$ ). Leaky modes have a small imaginary part (small leakage) and are clearly distinguished from PML modes. The criterion given by Eq. (16) allows an efficient and straightforward separation of leaky and PML modes.

\section{F. Remarks on mode normalization}

From a theoretical point of view, the exponential growth of leaky modes raises normalization issues. As proved in optics (see, e.g., [15]), a PML solves this intricate problem by transforming the divergent field of leaky modes into a decaying field.

Actually, a PML provides normalization and orthogonality for both leaky resonances and PML modes. Considering Eq. (12) and owing to the symmetry of the complex-valued stiffness and mass matrices, the following orthogonality relationships holds:

$$
\begin{gathered}
\hat{\mathbf{U}}_{l}^{(j) \mathrm{T}} \mathbf{K}(l) \hat{\mathbf{U}}_{l}^{(i)}=\omega_{l}^{(i) 2} \delta_{i j}, \\
\hat{\mathbf{U}}_{l}^{(j) \mathrm{T}} \mathbf{M}(l) \hat{\mathbf{U}}_{l}^{(i)}=\delta_{i j},
\end{gathered}
$$

where the superscripts $m$ have been dropped for conciseness of notations (and since the eigensystem is degenerate with respect to $m$ ). Based on modal expansions, these orthogonality relationships can be used to compute the forced response of spheres to optimize the generation of WGMs. Details about this type of calculation can be found in Ref. [22] for in vacuo spheres (i.e., without PML). With a PML, the result remains unchanged because the relationships (18) still hold in both cases. For paper conciseness, the presentation of the computation process of the forced response is not repeated here.

\section{RESULTS}

This section presents numerical results computed with the semianalytical FE formulation. In particular, we investigate the high-frequency behavior of leaky WGMs in embedded spheres.

As outlined in the introduction, it is of importance to take into account the material loss. For simplicity, we will consider a viscoelastic frequency independent hysteretic model. With this model, the bulk wave velocities $\tilde{c}_{l}$ and $\tilde{c}_{s}$ of the materials are complex and given by

$$
\tilde{c}_{l, s}=c_{l, s}\left(1+\mathrm{i} \frac{\kappa_{l, s}}{2 \pi}\right)^{-1},
$$

where $\kappa_{l}$ and $\kappa_{s}$ denote the bulk wave attenuations in nepers per wavelength.

Two test cases are considered. The first case is that of a stiff sphere, that is, a sphere material with bulk wave velocities greater than the external medium. The second test case is that of a soft sphere (bulk wave velocities lower than the external medium). Results computed for embedded spheres are compared with those for in vacuo spheres. A thorough investigation of these configurations allows the identification of general trends about the behavior of leaky WGMs. 
TABLE I. Material properties.

\begin{tabular}{lccccc}
\hline \hline Material & $\rho\left(\mathrm{kgm}^{-3}\right)$ & $c_{l}\left(\mathrm{~ms}^{-1}\right)$ & $c_{s}\left(\mathrm{~ms}^{-1}\right)$ & $\kappa_{l}\left(\mathrm{~Np} \lambda^{-1}\right)$ & 0.003 \\
\hline Steel $^{\mathrm{a}}$ & 7932 & 5500.7 & 3175.8 & 0.186 & 0.008 \\
Concrete $^{\mathrm{b}}$ & 2152 & 3758 & 2090 & 0.029 & 0.229 \\
Epoxy $^{\mathrm{c}}$ & 1600 & 2960 & 1450 & 0.043 \\
\hline \hline
\end{tabular}

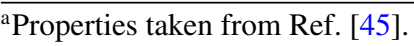

${ }^{\mathrm{b}}$ Properties taken from Ref. [46].

${ }^{\mathrm{c}}$ Properties taken from Ref. [47].

\section{A. Model parameters}

A concrete medium is chosen for the embedment. The sphere is made of steel in the stiff case and epoxy in the soft case. Material parameters are given in Table I. A perfectly bonded interface is assumed between the sphere and the external matrix (i.e., continuity of stress and displacement in the three directions).

The PML parameters are set to $h=0.25 a, \hat{\gamma}=1+2 i$. This choice follows from a convergence study (not shown for conciseness), by varying both $h$ and $\hat{\gamma}$. The radial coordinate is meshed with one-dimensional quadratic FE of length $0.005 a$, yielding 1500 degrees of freedom (dofs) (reduced to 1200 dofs in the in vacuo case). The eigenproblem (12) is solved repeatedly for a wide range of wave numbers, $l=$ $0, \ldots, 150$. In order to compute mainly high-frequency leaky modes, the normalized eigenvalues $\omega a / c_{s_{0}}$ are solved around a user-defined shift equal to $1.2 l$ in the eigenvalue solver (see ARPACK library [48]).

Dispersion curves will be given as a function of $l$ for the phase velocity $v_{p}$, the group velocity $v_{g}$ [30], and the $Q$ factor, defined by

$$
v_{p}=\frac{\operatorname{Re}\left(\omega_{l}^{(n)}\right) a}{l+1 / 2}, v_{g}=\operatorname{Re}\left(\frac{\partial \omega_{l}^{(n)}}{\partial l}\right) a, Q=-\frac{\operatorname{Re}\left(\omega_{l}^{(n)}\right)}{2 \operatorname{Im}\left(\omega_{l}^{(n)}\right)} .
$$

The group velocity can be postprocessed as follows (see Ref. [22] for proof):

$$
v_{g}=\operatorname{Re}\left\{\frac{\hat{\mathbf{U}}_{l}^{(n) \mathrm{T}}\left(\frac{\partial \mathbf{K}(l)}{\partial l}-\omega_{l}^{(n) 2} \frac{\partial \mathbf{M}(l)}{\partial l}\right) \hat{\mathbf{U}}_{l}^{(n)}}{2 \omega_{l}^{(n)} \hat{\mathbf{U}}_{l}^{(n) \mathrm{T}} \mathbf{M}(l) \hat{\mathbf{U}}_{l}^{(n)}}\right\} a .
$$

Depending on the configuration, the parameter $\eta_{\min }$ for filtering PML modes has been set between 0.8 and 0.9 .

\section{B. Case 1: Stiff-sphere configuration}

Figure 5 shows the dispersion curves of spheroidal modes for the stiff configuration (steel into concrete), as well as for the in vacuo steel sphere. The dispersion curves for torsional modes are shown in Fig. 6.

As a general trend, the phase velocity tends toward $c_{s_{0}}$, the shear wave bulk velocity of the sphere, as $l$ increases (i.e., in the high-frequency regime), for all modes except the fundamental spheroidal modes $n=0$ (this particular mode will be discussed later). This velocity limit can be more clearly observed for the group velocity. WGMs have hence a nondispersive behavior as the frequency increases. Besides, both phase and group velocities with the embedment remain close to their in vacuo counterparts.

Compared to torsional modes [Fig. 6(b)], the nonmonotonic changes observed in the group velocities of spheroidal modes [Fig. 5(b)] can be explained by the complex body wave combination of $\mathrm{P}$ waves and SV waves traveling through the sphere, reflected and converted several times at the surface, and fulfilling constructive interference conditions [5,44]. As the polar wave number $l$ increases, the spheroidal modes become mainly formed from a combination of multiple reflected SV waves (the polar displacement $u_{\theta}$ nearly goes to zero), which explains why they tend toward a shear wave behavior. These modes are the so-called transverse spheroidal modes, observed for a sphere in vacuum [8], for which the $\mathrm{SV}$-wave content prevails. Compared to spheroidal modes, torsional modes rather tend monotonically toward the shear wave behavior because torsional modes are formed from multiple reflected horizontal shear waves ( $\mathrm{SH}$ waves) only (no conversion) $[5,44]$.

The $Q$ factor behaves completely differently for the embedded sphere [see Figs. 5(c) and 6(c)]. In vacuo, the $Q$ factor of spheroidal modes quickly decreases toward $Q_{s_{0}}=$ $\pi / \kappa_{s_{0}}$, the $Q$ factor of shear waves inside the sphere. The $Q$ factor of torsional modes remains constant and equal to $Q_{s_{0}}$. Conversely, with embedment, the $Q$ factor is weak in the low-frequency regime and then slowly increases in the high-frequency regime, up to $Q_{s_{0}}$ according to the shear wave limit.

These results are consistent with the competing effects of viscoelasticity and leakage found in embedded elastic waveguides [45]: as the frequency increases, the attenuation due to viscoelasticity tends to increase while the attenuation due to leakage tends to decrease. Note that if viscoelasticity was neglected $\left(\kappa_{s} \rightarrow 0\right), Q$ would tend to infinity in the high-frequency regime, which would be unrealistic (hence the importance of taking into account material loss).

In a stiff configuration, the $Q$ factor tends to improve as the mode order $n$ grows. This trend is particularly visible for torsional modes, although less significant for spheroidal modes. It can be explained by the energy distribution, concentrated near the interface for low-order modes, hence increasing their leakage in the external medium.

As far as the fundamental spheroidal mode $n=0$ is concerned (see Fig. 5), its in vacuo characteristics tend toward those of the Rayleigh wave in the high-frequency regime. In a half space, the Rayleigh wave speed can be approximated by $\tilde{c}_{r_{0}} \simeq \tilde{c}_{s_{0}}\left(0.862+1.14 \tilde{v_{0}}\right) /\left(1+\tilde{v_{0}}\right)$ [where $\tilde{v_{0}}=0.5\left(\tilde{c}_{l_{0}}^{2}-\right.$ $\left.2 \tilde{c}_{s_{0}}^{2}\right) /\left(\tilde{c}_{l_{0}}^{2}-\tilde{c}_{s_{0}}^{2}\right)$ is the complex Poisson ratio], yielding a quality factor roughly equal to $Q_{r_{0}} \simeq-0.5 \operatorname{Im}\left(\tilde{c}_{r_{0}}\right) / \operatorname{Re}\left(\tilde{c}_{r_{0}}\right)$. 

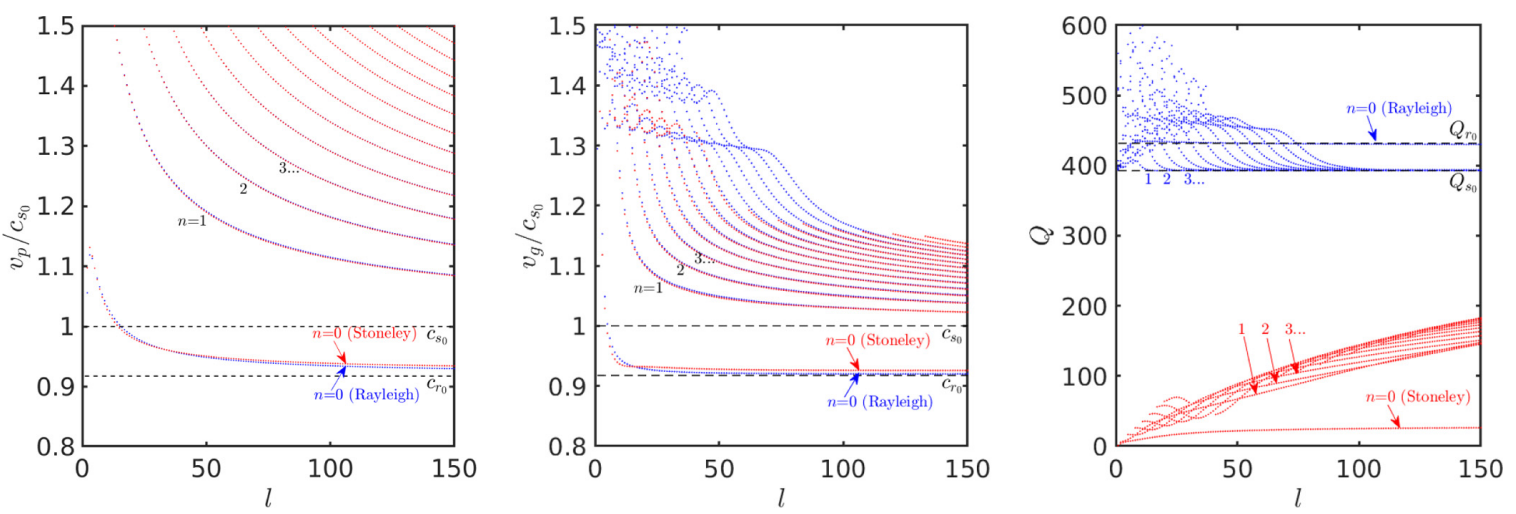

FIG. 5. Stiff-sphere configuration, spheroidal modes. From left to right: Phase velocity, group velocity, and $Q$ factor as a function of the polar wave number $l\left(\eta_{\min }=0.8\right)$. Red: Steel into concrete; blue: steel in vacuum.

Embedded in concrete, the fundamental spheroidal mode corresponds to a solid-solid interface wave, which hence tends toward the behavior of a Stoneley wave between two elastic half spaces. Note that its phase velocity remains higher than the shear bulk wave velocities so that this Stoneley wave is of leaky type (attenuating). The $Q$ factor of the $n=0$ Stoneleylike wave is very low compared to the other modes. Such a wave hence appears of less interest for generating WGMs.

The main conclusion is that the $Q$ factor of leaky WGMs cannot exceed $\pi / \kappa_{s_{0}}$, the quality factor of shear waves inside the material constituting the sphere $(\simeq 400$ for steel). The $Q$ factor reached by elastic WGMs is hence far weaker than in optics $\left(Q \approx 10^{9}\right.$ for $l \approx 1000$ in practice).

Additional numerical tests have been conducted to further explore the behavior of leaky WGMs (results not shown for conciseness). By increasing the contrast between materials, the convergence of the $Q$ factor toward $Q_{s_{0}}$ for large $l$ becomes faster (this has been observed by artificially multiplying the density of steel by 10). As far as Stoneley waves are concerned, their behavior is more complex than Rayleigh waves. Their existence depends on material combination. This has been checked by artificially dividing the density of steel by 10. In this case, no Stoneley-like mode has been found. This is consistent with the theoretical findings of Ref. [49] (the Stoneley wave disappears if the material of greater density has a velocity much lower).

\section{Case 2: Soft-sphere configuration}

A soft-sphere configuration offers a stronger analogy with WGMs in optics. In optics, total internal reflection can be obtained when light propagates faster in the embedding medium than in the sphere. In elasticity, total internal reflection is more complicated to obtain because of multiple reflection, transmission, and conversion of three kinds of waves $(\mathrm{P}, \mathrm{SV}$, and SH waves) [50]. Yet, we can expect a behavior similar to light when both shear wave and longitudinal wave velocities are smaller in the sphere than in the embedding medium, that is, in the case of a soft inclusion into a stiffer matrix.

For a steel sphere (stiff configuration), there was a critical angle only for the conversion of SV waves into $\mathrm{P}$ waves, therefore leading to significant leakage loss and a slow increase of the $Q$ factor. Let us replace steel with epoxy (soft configuration). The shear and longitudinal bulk waves of epoxy are slower than in the embedding medium, so that both waves indeed admit critical angles in reflection [50] (although P-wave reflection into a reflected SV wave can still occur).

Figure 7 shows the dispersion curves of spheroidal modes for the soft configuration, as well as for the in vacuo epoxy sphere. The dispersion curves for torsional modes are shown in Fig. 8.

Several differences can be observed compared to the stiff configuration. First, no Stoneley-like wave is found for this material combination (as shown in Fig. 7, a Rayleigh wave is
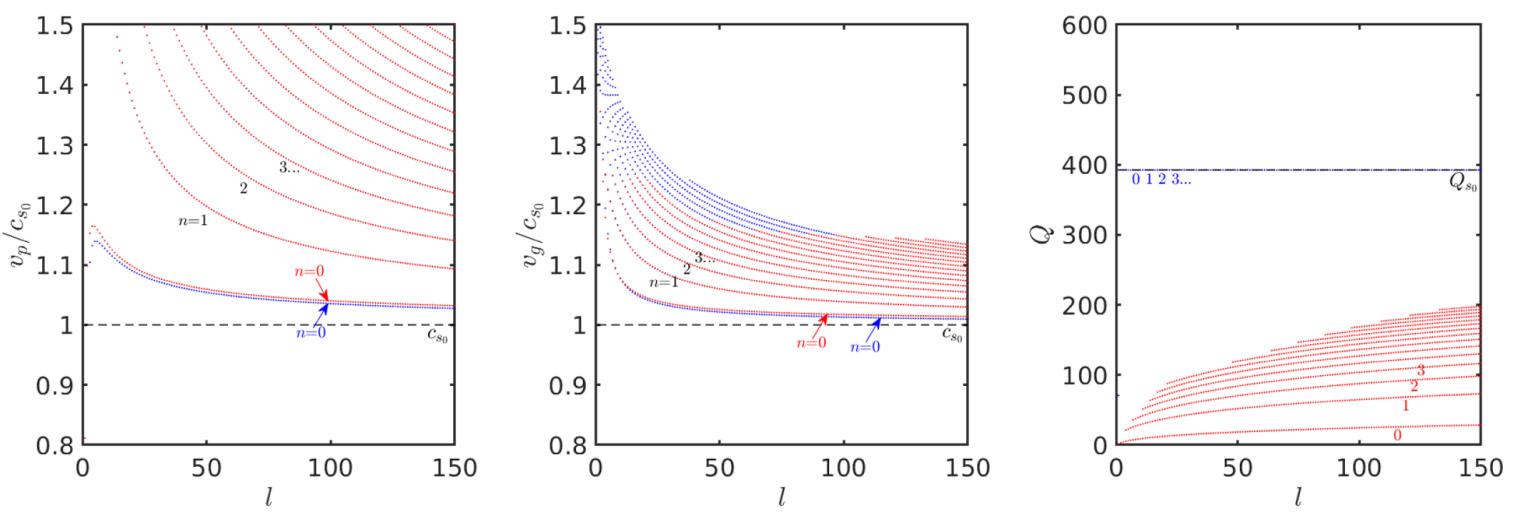

FIG. 6. Same caption as Fig. 5 but for torsional modes. 

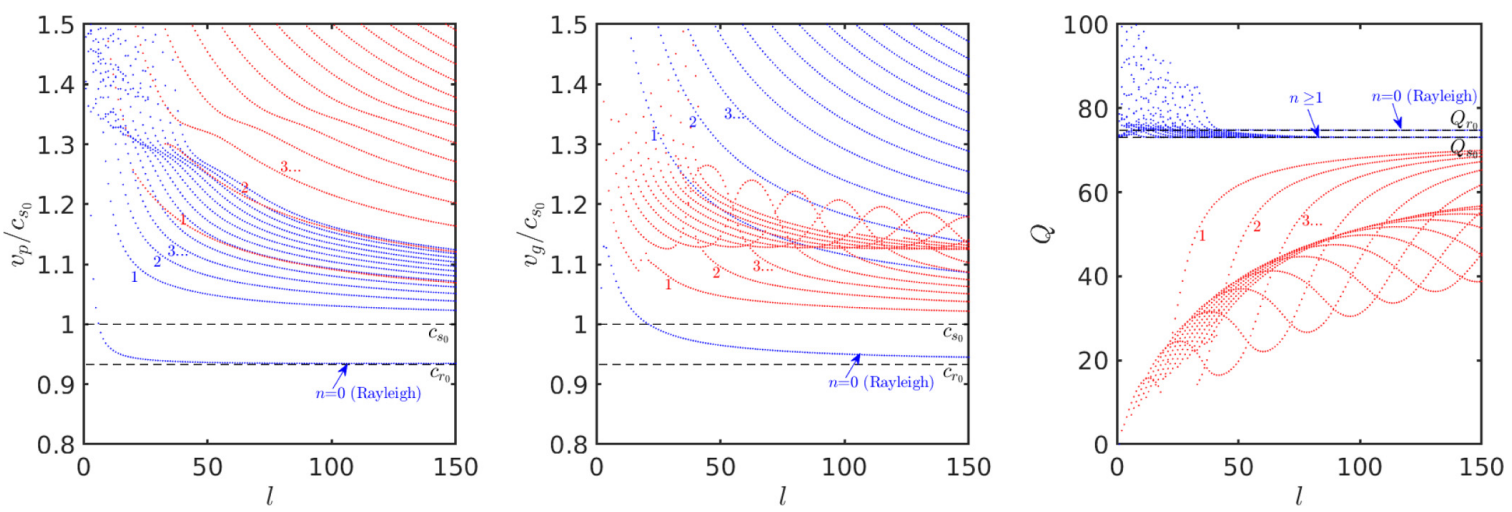

FIG. 7. Soft-sphere configuration, spheroidal modes. From left to right: Phase velocity, group velocity, and $Q$ factor as a function of the polar wave number $l\left(\eta_{\min }=0.8\right)$. Red: Epoxy into concrete; blue: epoxy in vacuum.

found in vacuo but no Stoneley wave is found in the embedded sphere). Second, the velocities (both phase and group) are significantly different from their in vacuo counterparts. Third, the $Q$ factor tends toward $Q_{s_{0}}$ far more rapidly than in the stiff configuration. Fourth, for a given $l$, the $Q$ factor tends to decrease as the mode order $n$ increases (as opposed to the stiff configuration). This behavior is quite similar to those of WGMs in optics [1,51].

As a side remark, it can be observed that the group velocity of spheroidal modes [Fig. 7(b)] changes in a strong nonmonotonic manner, with maxima of group velocities coinciding with minima of $Q$ factors (this phenomenon also occurs in Fig. 5 but is less visible). These maxima correspond to a polarization of spheroidal modes in the azimuthal direction. Such modes correspond to the so-called pseudolongitudinal modes of a sphere in vacuum [8], for which the P-wave contribution is significant. The $Q$ factor of pseudolongitudinal modes and torsional modes strongly depends on the bonding condition of the interface, as will be briefly discussed in Sec. III D.

The main result with this test case is that the $Q$ factor can quickly tend toward $Q_{s_{0}}$ in a soft-sphere configuration. However, this advantage is counterbalanced by the fact that quality factors are usually significantly weaker in soft than in stiff materials in practice $\left(Q_{s_{0}}<100\right.$ for epoxy).

\section{Perfectly sliding interface}

To further illustrate the influence of the polarization of WGMs, we consider the case of a perfectly sliding interface. Only the continuity of normal stress and normal displacement is therefore allowed at the interface between the sphere and the external medium. The tangential components of the displacement, $u_{\theta}$ and $u_{\phi}$, are free.

With a sliding interface, note that the torsional modes are no longer coupled to the external medium. Their characteristics are exactly the same as in vacuo (in particular, the $Q$ factor of torsional modes remains constant and equal to $Q_{s_{0}}$ ); see blue curves in Figs. 6 and 8.

Conversely, the behavior of spheroidal modes is strongly affected. Figures 9 and 10 show the $Q$ factor computed with a sliding interface in the stiff and soft configurations, respectively. As opposed to a perfectly bonded interface, the $Q$ factor can reach high values in a low-frequency range. These values appear to be close to the Rayleigh $Q$ factor. This striking behavior can be explained by the polarization of spheroidal modes in the low-frequency regime. As mentioned previously, spheroidal modes in this frequency regime correspond to pseudolongitudinal modes, mainly oriented along the azimuthal direction, which minimizes their sensitivity to the external medium.
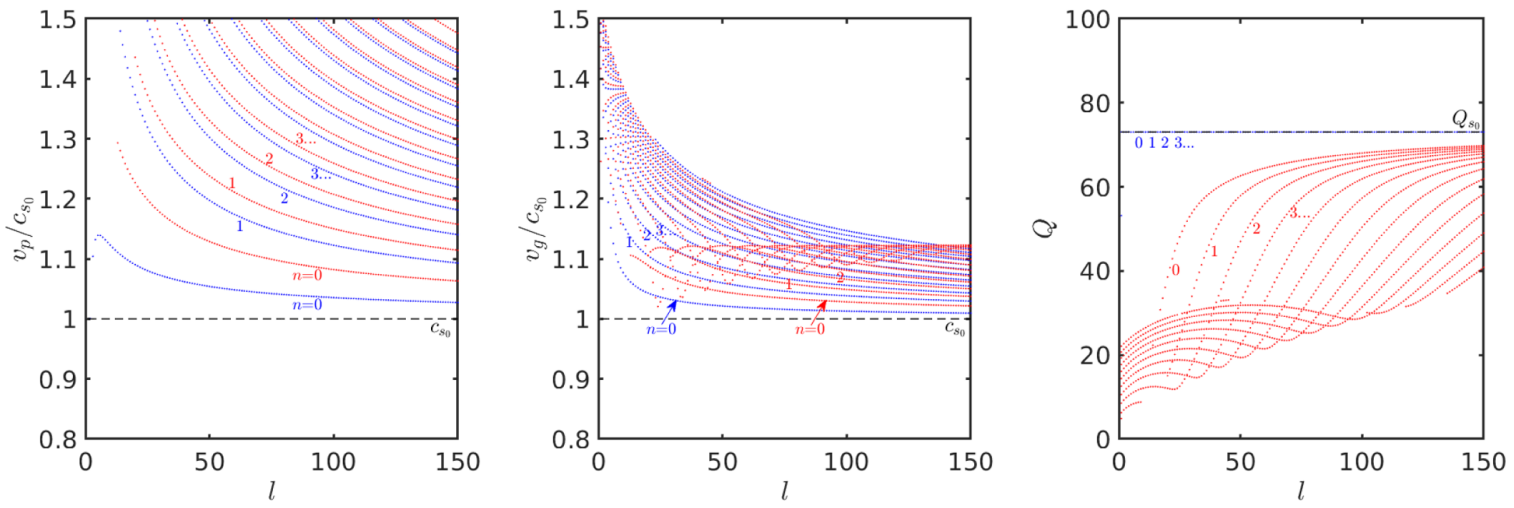

FIG. 8. Same caption as Fig. 7 but for torsional modes $\left(\eta_{\min }=0.9\right)$. 


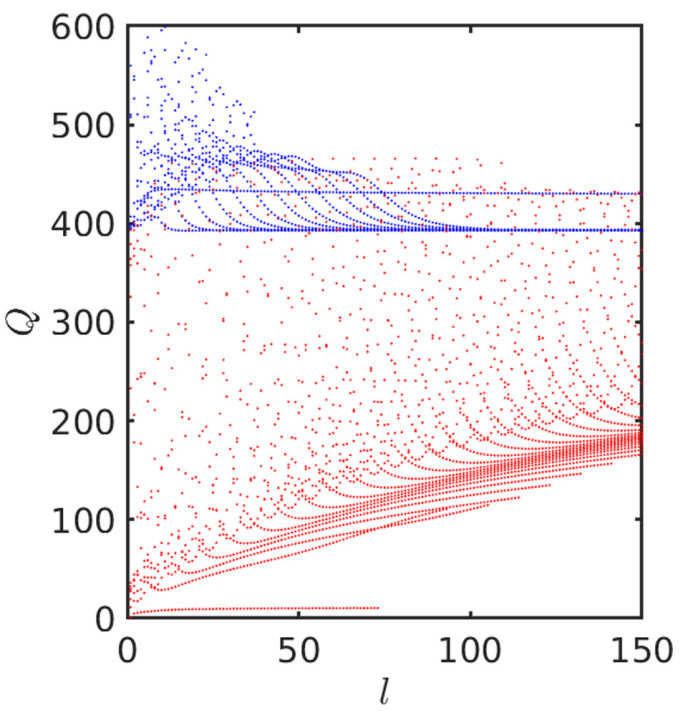

FIG. 9. Same caption as Fig. 5 but for a sliding interface (for conciseness, only the $Q$ factor of spheroidal modes is shown).

\section{CONCLUSION}

In this paper, we have investigated the characteristics of high-frequency whispering-gallery modes in embedded elastic spheres. For this purpose, a specific FE method in spherical coordinates has been proposed. With this method, only the radial coordinate is discretized while the angular distribution of elastodynamic fields is described analytically. Besides, a radial PML is introduced to cope with the unbounded nature of the problem. Our approach leads to a linear matrix eigensystem, simple and fast to solve in the high-frequency regime. The solution of the eigenproblem delivers leaky and PML modes. The leaky modes are revealed thanks to the PML, by the rotation of the continuum in the complex frequency plane, allowing access to the improper Riemann sheets. The PML modes, related to the continuum of radiation modes, are not

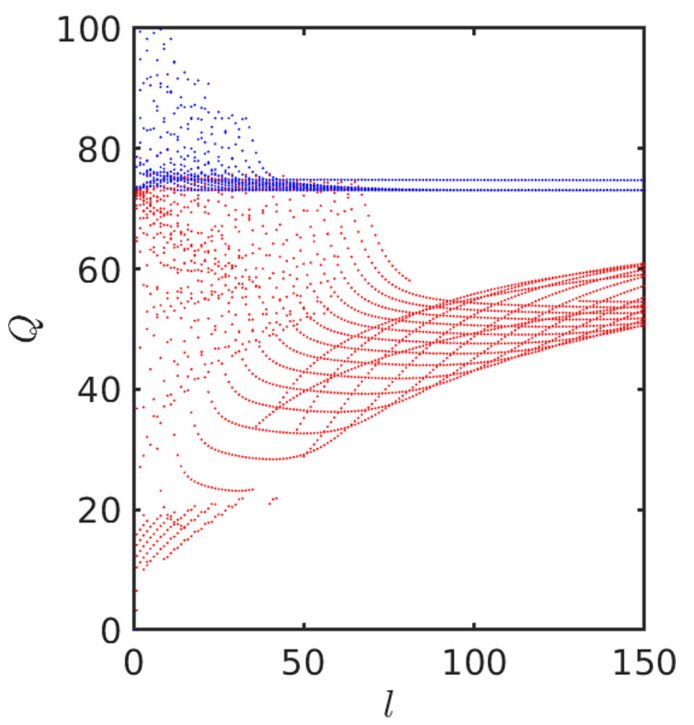

FIG. 10. Same caption as Fig. 7 but for a sliding interface (for conciseness, only the $Q$ factor of spheroidal modes is shown). intrinsic to the physics but can be efficiently filtered out, in a postprocessing step, thanks to an energy-based criterion. Besides, the PML can provide normalization and orthogonality for the leaky modes, which allows the computation of forced responses based on modal expansions.

Two configurations have been considered, corresponding to a stiff sphere and a soft sphere. Material loss has been included by considering a viscoelastic hysteretic model. Our results highlight the behavior of leaky elastic WGMs in the high-frequency regime, not yet considered in the literature. Except for the Stoneley-like fundamental spheroidal mode (peculiar to elasticity but of low $Q$ factor), the modal characteristics of leaky WGMs tends, as the frequency increases, toward the shear wave properties of the material constituting the sphere. As opposed to leakage, the material viscoelasticity tends to increase the attenuation of waves with frequency, leading to relatively low $Q$ factor limits.

In particular, it has been shown that the $Q$ factor of leaky WGMs tends to slowly increase up to the quality factor of the shear bulk waves of the sphere. Owing to internal reflection mechanisms and by analogy with optics, this slow increase can be enhanced in the case of a soft-sphere configuration. However, this advantage is counterbalanced by the fact that quality factors are usually weaker in soft than in stiff materials.

Elastic WGMs in spheres could be of prime interest for sensing the mechanical properties of an external medium, e.g., by measuring a shift of their eigenfrequency [52]. More generally, we expect that elastic WGMs could be optimized in a more complex configuration, considering multilayered spheres for instance. The formulation proposed in this paper is versatile and allows modeling such complex spheres without difficulties.

\section{ACKNOWLEDGMENTS}

This work has received support under the program "Investissement d'Avenir" launched by the French Government and implemented by ANR, with the reference ANR-16-IDEX0003, under the program "Paris scientifiques" of Région Pays de la Loire (Project SMOg). M.G. thanks Dr. C. Hazard (ENSTA, POEMS, Palaiseau, France) for discussions about the Rellich's lemma. F.T. wishes to thank V. Pagneux (Le Mans Université, LAUM, France) for helpful suggestions about the manuscript.

\section{APPENDIX: FE MATRICES}

In this Appendix, we give the detailed expression of the FE matrices involved in Eq. (12).

The elementary mass matrix is given by

$$
\mathbf{M}^{e}(l)=\int \rho \mathbf{N}^{e \mathrm{~T}}\left[\begin{array}{lll}
1 & 0 & 0 \\
0 & \bar{l} & 0 \\
0 & 0 & \bar{l}
\end{array}\right] \mathbf{N}^{e} \tilde{r}^{2} \gamma d r .
$$

The stiffness matrix can be written as

$$
\mathbf{K}(l)=\mathbf{K}_{1}(l)+\mathbf{K}_{2}(l)+\mathbf{K}_{2}^{\mathrm{T}}(l)+\mathbf{K}_{3}(l),
$$


641

$$
\mathbf{K}_{1}^{e}(l)=\int \frac{d \mathbf{N}^{e \mathrm{~T}}}{d r}\left[\begin{array}{ccc}
C_{11} & 0 & 0 \\
0 & \bar{l} C_{55} & 0 \\
0 & 0 & \bar{l} C_{55}
\end{array}\right] \frac{d \mathbf{N}^{e}}{d r} \frac{\tilde{r}^{2}}{\gamma} d r
$$

$$
\mathbf{K}_{3}^{e}(l)=\int \mathbf{N}^{e \mathrm{~T}}\left[\begin{array}{ccc}
\bar{l} C_{55}+4\left(C_{23}+C_{44}\right) & -\bar{l}\left[C_{55}+2\left(C_{23}+C_{44}\right)\right] & 0 \\
-\bar{l}\left[C_{55}+2\left(C_{23}+C_{44}\right)\right] & \bar{l}^{2} C_{23}+\bar{l} C_{55}+2 \bar{l}(\bar{l}-1) C_{44} & 0 \\
0 & 0 & \bar{l} C_{55}+\bar{l}(\bar{l}-2) C_{44}
\end{array}\right] \mathbf{N}^{e} \gamma d r
$$

The above expressions differ from those of Ref. [22] due to the introduction of the PML, which yields complex-valued matrices. Without PML $(\gamma=1, \tilde{r}=r)$, the expressions well degenerate to the in vacuo case treated in [22].
[1] A. N. Oraevsky, Quantum Electron. 32, 377 (2002).

[2] M. R. Foreman, J. D. Swaim, and F. Vollmer, Adv. Opt. Photonics 7, 168 (2015).

[3] H. Lamb, Proc. London Math. Soc. 1, 189 (1881).

[4] Y. Sato and T. Usami, Geophys. Mag. 31, 15 (1962).

[5] K. Aki and P. G. Richards, Quantitative Seismology: Theory and Methods, Vol. 1 (W. H. Freeman, 1980).

[6] L. Saviot, D. B. Murray, and M. C. Marco de Lucas, Phys. Rev. B 69, 113402 (2004).

[7] L. Saviot and D. B. Murray, Phys. Rev. B 72, 205433 (2005).

[8] B. Sturman and I. Breunig, J. Appl. Phys. 118, 013102 (2015).

[9] Y. Shin, Y. Liu, M. Vomir, and J.-W. Kim, Phys. Rev. B 101, 020302(R) (2020).

[10] N. G. Einspruch, E. Witterholt, and R. Truell, J. Appl. Phys. 31, 806 (1960).

[11] J.-P. Sessarego, J. Sageloli, R. Guillermin, and H. Überall, J. Acoust. Soc. Am. 104, 2836 (1998).

[12] P. Verma, W. Cordts, G. Irmer, and J. Monecke, Phys. Rev. B 60, 5778 (1999).

[13] C. Voisin, D. Christofilos, N. Del Fatti, and F. Vallée, Phys. B: Condens. Matter 316, 89 (2002).

[14] D. B. Murray and L. Saviot, Phys. Rev. B 69, 094305 (2004).

[15] P. Lalanne, W. Yan, K. Vynck, C. Sauvan, and J.-P. Hugonin, Laser Photonics Rev. 12, 1700113 (2018).

[16] M. Mansuripur, M. Kolesik, and P. Jakobsen, Phys. Rev. A 96, 013846 (2017).

[17] V. Dubrovskiy and V. Morochnik, Izv., Acad. Sci., USSR, Phys. Solid Earth (Engl. Transl.) 17, 494 (1981).

[18] K. L. J. Fong, A study of curvature effects on guided elastic waves, Ph.D. thesis, University of London, 2005.

[19] G. R. Buchanan and G. R. Ramirez, J. Sound Vib. 253, 724 (2002).

[20] P. R. Heyliger and A. Jilani, Int. J. Solids Struct. 29, 2689 (1992).

[21] J. Park, Wave motion in finite and infinite media using the thin-layer method, Ph.D. thesis, Massachusetts Institute of Technology, 2002.

[22] M. Gallezot, F. Treyssède, and O. Abraham, Wave Motion 96, 102555 (2020).

[23] Z. Martinec, Geophys. J. Int. 142, 117 (2000).

[24] W. C. Chew and W. H. Weedon, Microwave Opt. Technol. Lett. 7, 599 (1994).
[25] S. Hein, T. Hohage, and W. Koch, J. Fluid Mech. 506, 255 (2004).

[26] S. Kim and J. E. Pasciak, Math. Comput. 78, 1375 (2009).

[27] B. Vial, F. Zolla, A. Nicolet, and M. Commandré, Phys. Rev. A 89, 023829 (2014).

[28] W. Yan, R. Faggiani, and P. Lalanne, Phys. Rev. B 97, 205422 (2018).

[29] K. Bathe, Finite Element Procedures (Prentice-Hall, Englewood Cliffs, 1995).

[30] A. C. Eringen and E. S. Şuhubi, Elastodynamics, Vol. II (Academic Press, 1975).

[31] E. Kausel, Fundamental Solutions in Elastodynamics: A Compendium (Cambridge University Press, 2006).

[32] G. B. Arfken and H. J. Weber, Mathematical Methods for Physicists (Academic Press, 1999).

[33] A. Silbiger, J. Acoust. Soc. Am. 34, 862 (1962).

[34] J. C. Knight, N. Dubreuil, V. Sandoghdar, J. Hare, V. LefèvreSeguin, J. M. Raimond, and S. Haroche, Opt. Lett. 20, 1515 (1995).

[35] P. Malischewsky, Surface Waves and Discontinuities (Elsevier, 1987).

[36] R. Collin, Field Theory of Guided Waves, 2nd ed. (IEEE Press, New York, 1991).

[37] M. Gallezot, F. Treyssède, and L. Laguerre, J. Acoust. Soc. Am. 141, EL16 (2017).

[38] F. Rellich, Jahresbericht der Deutschen MathematikerVereinigung 53, 57 (1943).

[39] H. Überall, Phys. Acoust. 10, 1 (1973).

[40] F. Treyssède, K. Nguyen, A.-S. Bonnet-Ben Dhia, and C. Hazard, Wave Motion 51, 1093 (2014).

[41] H. Derudder, F. Olyslager, D. D. Zutter, and S. V. den Berghe, IEEE Trans. Antennas Propag. 49, 185 (2001).

[42] M. Gallezot, F. Treyssède, and L. Laguerre, J. Comput. Phys. 356, 391 (2018).

[43] M. Gallezot, F. Treyssède, and L. Laguerre, J. Sound Vib. 443, 310 (2019).

[44] J. N. Brune, J. Geophys. Res. 71, 2959 (1966).

[45] B. Pavlakovic, M. J. S. Lowe, and P. Cawley, J. Appl. Mech. 68, 67 (2001).

[46] A. Marzani, E. Viola, I. Bartoli, F. L. di Scalea, and P. Rizzo, J. Sound Vib. 318, 488 (2008). 
[47] J. Barshinger and J. Rose, IEEE Trans. Ultrason. Ferroelectr. Freq. Control 51, 1547 (2004).

[48] R. B. Lehoucq, D. C. Sorensen, and C. Yang, ARPACK Users' Guide: Solution of Large-Scale Eigenvalue Problems with Implicitly Restarted Arnoldi Methods, Vol. 6 (SIAM, 1998).

[49] W. L. Pilant, Bull. Seismol. Soc. Am. 62, 285 (1972).
[50] B. A. Auld, Acoustic Fields and Waves in Solids, Vol. 2 (WileyInterscience, 1973).

[51] A. Aiello, J. G. E. Harris, and F. Marquardt, Phys. Rev. A 100, 023837 (2019).

[52] V. Ilchenko, P. Volikov, V. Velichansky, F. Treussart, V. LefevreSeguin, J.-M. Raimond, and S. Haroche, Opt. Commun. 145, 86 (1998). 